\title{
Comparison of Reproductive Outcome of Falloposcopic Tuboplasty for the Proximal Tubal Obstruction with or without Chlamydia Trachomatis Infection
}

\author{
Hiroshi Nabeshima ${ }^{*}, 1$, Takashi Murakami ${ }^{2}$, Shinichi Hayasaka ${ }^{2}$, Yukihiro Terada ${ }^{2}$, Nobuo Yaegashi ${ }^{2}$ \\ and Kunihiro Okamura ${ }^{2}$ \\ ${ }^{I}$ Department of Obstetrics and Gynecology, Iwate Prefectural Iwai Hospital, Iwate, Japan \\ ${ }^{2}$ Department of Obstetrics and Gynecology, Tohoku University Graduate School of Medicine, Miyagi, Japan
}

\begin{abstract}
Chlamydia trachomatis (CT) infection is a common cause of tubal factor infertility, and is considered a contraindication for tubal surgery. In vitro fertilization with embryo transfer (IVF/ET) is the most widely used treatment for tubal factor infertility, including that caused by CT infection. Falloposcopy to re-canalize occluded tubes may also be useful therapeutically. It is unclear, however, what effect CT infection has on its efficacy. We examined the relationship between the efficacy of Falloposcopic tuboplasty (FT) and CT infection, retrospectively. Of 38 patients with tubal obstruction in which the surgery was performed, with follow-up, 21 patients had CT infections while 17 patients were not infected. In the Chlamydia positive group $(\mathrm{CT}+), 38$ tubes were treated with FT and 29 tubes were treated in the Chlamydia negative group (CT-). Re-canalization rates per tube was $60 \%$ in the $\mathrm{CT}+$ group and $79 \%$ in the $\mathrm{CT}$ - group. The success rate per case was $90 \%$ in the CT+ group and $94 \%$ in the CT- group. Cases were followed-up using either hysterosalpingography or second look laparoscopy, and a post-operative patency rate of $56 \%$ in the CT+ group and $75 \%$ in the CT- group was observed. In patients in followed for two years or more, the CT+ group had 5 spontaneous pregnancies (36\%) and the CTgroup had 9 spontaneous pregnancies $(60 \%)$. There was a trend to better outcome in the CT- group, but there was no significant statistical difference between the two groups. In Conclusion, CT infection decreases fertility after FT. Despite CT infection having a negative impact on FT, the CT+ group had a post-operative pregnancy rate of $35.7 \%$, which was comparable to IVF-ET. Given this, we recommend FT for patients who have isolated tubal occlusion before beginning IVF/ET, even if the patients were previously infected with CT.
\end{abstract}

Keywords: Falloposcopy, falloposcopic tuboplasty, tubal obstruction, chlamydia trachomatis, PID.

\section{INTRODUCTION}

Chlamydia trachomatis (CT) infection is a common cause of tubal factor infertility, and is considered a contraindication for tubal surgery [1]. In vitro fertilization with embryo transfer (IVF/ET) is the most widely used treatment for tubal factor infertility, including that caused by CT infection. Falloposcopy to re-canalize occluded tubes may also be useful therapeutically [2]. It is unclear, however, what effect CT infection has on its efficacy. In this report, we examined the relationship between fertility outcome and pre-operative CT infection.

\section{MATERIAL AND METHOD}

Thirty eight patients were included in this study. All had bilateral proximal tubal obstruction diagnosed by hysterosalpingography (HSG). All underwent Falloposcopic tuboplasty (FT) between December 1995 and November 2002 in Tohoku University hospital.

CT infection status was ascertained in all patients either with serological testing for CT IgA antibody and/or the presence of cervical CT antigen prior to surgery. The patients were classified into two groups based on whether or not they had evidence of Chlamydia infection. One patient whose partner had a sperm factor was excluded from this report.

*Address correspondence to this author at the Department of Obstetrics and Gynecology, Iwate Prefectural Iwai Hospital, 17, Ohira, Kozenji, Ichinoseki-city, Iwate, 029-0192, Japan; Tel: 81-191-23-3452; Fax: 81-191-239691; Email: nabe-akt@umin.ac.jp and nabeji@hi-ho.ne.jp
The CT positive patients were treated with antibiotics for at least 2 weeks prior to surgery. The FT procedure was performed with laparoscopy under general anesthesia. In all patients, tubal occlusion was confirmed with indigo carmine dye hydrotubation under laparoscopy. The FT procedure was not used for false occlusion cases.

The FT procedure was performed using the linear everting (LE) catheter system (Terumo Corporation, Tokyo, Japan). The LE catheter system consisted of an outer catheter sheath and an inner body attached to a tubular, balloon-like rolling membrane as described previously [3, 4]. A high resolution Falloposcope, having an image bundle of 2000 pixels and an outer diameter of $0.5 \mathrm{~mm}$, was then passed through the central cannel of the LE catheter and connected to a video camera and light source.

The balloon of the LE catheter delivered the Falloposcope into the tubal lumen to a maximum length of $10 \mathrm{~cm}$. The LE catheter containing the Falloposcope was advanced forward and into the Fallopian tube after balloon expansion and performance of the sliding procedure. Irrigation with a physiological saline solution was delivered through the catheter by an irrigation pump.

The entire procedure was observed under laparoscopy, and the successful re-canalization was judged by the outflow of dye from the tubal end. Re-canalization of at least one tube constituted a successful case.

The treated patients were evaluated by second look laparoscopy or HSG after surgery and the patency of the tube 
checked. The pregnancy rate was calculated for patients who were followed up for two years or more.

We report the re-canalization rate per tube, rate of successful cases, post-operative patency rate per tubes, and postoperative pregnancy rate per cases.

This report was a retrospective study. Data was assembled from chart reviews, patient questionnaires and by a telephone interview. Data were compared with the chisquare exact test for categorical comparisons. A P value of $<$ 0.05 was considered statistically significant. All statistical analyses were performed using the statistical program StatView ${ }^{\circledR}$ for Macintosh (Version 4.5 StatView, Abacus Concepts, Inc.).

\section{RESULTS}

Of the 38 patients who underwent FT surgery with follow-up, 21 cases had a pre-operative CT infection and 17 cases did not (Table 1). The total number of treated tubes was 67 tubes in 38 patients. In the CT positive group $(\mathrm{CT}+)$, 38 tubes in 21 patients were treated with FT. In the CT negative group (CT-), 29 tubes in 17 patients were treated with FT (Table 1). The re-canalization rates per tube were $60 \%$ $(23 / 38)$ in the CT+ group, and 79\% (23/29) in the CT-group.

Table 1. Background of Cases

\begin{tabular}{|l|c|c|}
\hline & CT(-) Group & CT(+) Group \\
\hline \hline Cases & 17 patients & 21 patients \\
\hline Age & $32 \pm 1.0$ & $20 \pm 0.8$ \\
\hline $\begin{array}{l}\text { Primary or Secondary } \\
\text { Infertility }\end{array}$ & $\begin{array}{c}\text { Prim.12 cases, } \\
\text { Sec. } 5 \text { cases }\end{array}$ & $\begin{array}{c}\text { Prim.12 cases, } \\
\text { Sec. } 9 \text { cases }\end{array}$ \\
\hline Laparoscopic findings & 29 tubes & 38 tubes \\
\hline Number of occluded tubes & $3 / 17(17.6 \%)$ & $2 / 21(9.5 \%)$ \\
\hline Endometriosis $(+)$ & $6.8 \pm 2.3$ & $16.6 \pm 5.1$ \\
\hline ASRM Adhesion classification &
\end{tabular}

Successful cases were defined as those in which the tube was re-canalized on at least one side. The Success rate per case was 90\% (19/21) in the CT+ group and 94\% (16/17) in the CT-group.

Of those tubes evaluated postoperatively with HSG or second look laparoscopy, the postoperative patency rates were $56 \%(10 / 18)$ per tube in the CT+ group and $75 \%$ $(12 / 16)$ per tube in the CT-group (Table 2$)$.

We were able to follow 29 of the patients (14 cases of $\mathrm{CT}+$ and 15 cases of CT-) for two years or more. Of these patients, 5 in the $\mathrm{CT}+$ group $(35 \%)$ became pregnant without IVF/ET and 9 in the CT- group (60\%) became pregnant without IVF/ET (Table 2). Ectopic pregnancy occurred in one of the 5 cases $(20 \%)$ in the CT- Group and two of the 9 cases $(22 \%)$ in the CT-group. The difference in pregnancy rate between these groups was not, however, statistically significant. All of the non IVF/ET pregnancies in this study occurred within a two year period of the FT procedure. In the $\mathrm{CT}+$ group, almost all pregnancies except one had occurred between one and two years from surgery (Table 2). There were no natural pregnancies two years after the FT procedure in either group. $80 \%$ of pregnancies occurred between one and two years after surgery.

\section{DISCUSSION}

Falloposcopy is usually used diagnostically to assess the patency of the tubal lumen. It is a more reliable method than HSG to confirm proximal occlusion and to rule out the false positive cases [5-8]. Recently, FT has also proven to be a highly useful, minimally invasive novel treatment for tubal infertility [2].

CT infection has a well documented association with PID and subsequent infertility [1,9-11]. CT may produce an asymptomatic infection in the female genital tract which is associated with silent tubal infection and tubal damage. Indeed, several studies suggest that serologic evidence of Chlamydia infection is related to an increased risk of asymptomatic tubal disease [9, 12]. Whether the diagnosis and treatment of asymptomatic Chlamydia positive patients improves fecundity remains unsolved [13]. Thus, many gynecologists are pressured to choose IVF/ET tubal surgery for treating patients with tubal occlusion and a history of CT infection [14].

In this report, we examined the efficacy of FT in the setting of CT infection. CT infection is generally accepted as a contraindication to tubal surgery. Indeed, the CT + group had worse results that the CT- group in all areas studied. However, there were no significant differences between the two groups. The effect of CT infection on the pregnancy rate was also analyzed. Although the rate of successful FT recanalization per case, and the follow-up patency and post

Table 2. Post Surgical Results and Post Surgical Pregnancy Rate

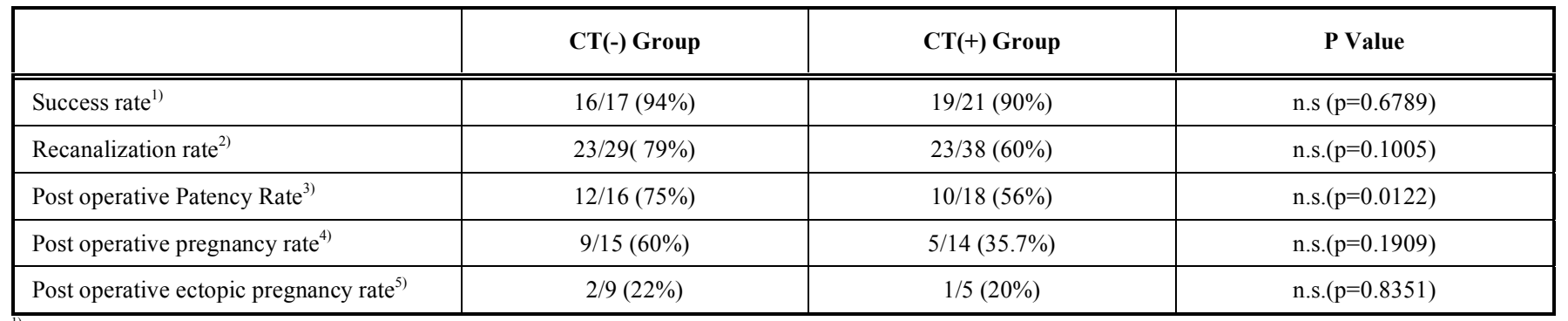

Success rate: The rate of cases in which recanalization was successful in one or both tubes.

${ }^{2)}$ Recanalization rate: successful recanalization per number of tubes attempted.

${ }^{3)}$ Post operative Patency Rate: Patency rate per recanalized tubes evaluated by follow up HSG or second look laparoscopy.

4) Post operative pregnancy rate: pregnancy rates of patients who were followed for six months or more among the cases in which recanalization was successful.

${ }^{5)}$ Post operative ectopic pregnancy rate: ectopic pregnancy cases among the post operative pregnancy group. 
operative non-IVF/ET pregnancy rates of the CT negative group were higher than in the positive group, there was no significant difference between the two groups. In the CT+ patients, the post-operative pregnancy rate was $35 \%$ and was comparable to that achieved by IVF/ET. There were no spontaneously pregnant patients two years after the FT procedure in either group, and $80 \%(4 / 5)$ of the pregnancies were concentrated between one and two years after surgery in the $\mathrm{CT}+$ group. The overall ectopic pregnancy rate was $22 \%$. This rate was very high. However, the rate between these groups was not statistically significant. We have been analyzing the data, and there is considerable correlation between pelvic adhesion score and ectopic pregnancy (the data was not shown in this report). Based on this study, we propose that $\mathrm{CT}+$ patients with proximal tubal occlusion should be treated with FT prior to undergoing IVF/ET for isolated tubal factor infertility. Ideally, the patient should then attempt become pregnant spontaneously for at least one to two years after surgery prior to moving to IVF.

\section{CONCLUSION}

This study shows that $\mathrm{CT}$ infection reduced the success rate of the FT procedure; however, this difference was not statistically significant. Therefore, we recommend FT as the first line treatment before IVF/ET, even in patients with tubal occlusion in the setting of CT infection.

\section{REFERENCES}

[1] Veenemans LM, van der Linden PJ. The value of Chlamydia trachomatis antibody testing in predicting tubal factor infertility. Hum Reprod 2002; 17: 695-698.

[2] Sueoka K, Asada H, Tsuchiya S, Kobayashi N, et al. Falloposcopic tuboplasty for bilateral tubal occlusion. A novel infertility treat- ment as an alternative for in-vitro fertilization?. Hum Reprod 1998; 13: 71-74.

[3] Pearlstone AC, Surrey ES, Kerin JF. The linear everting catheter: a nonhysteroscopic, transvaginal technique for access and microendoscopy of the fallopian tube. Fertil Steril 1992; 58: 854-857.

[4] Scudamore IW, Dunphy BC, Cooke ID. Outpatient falloposcopy: intra-luminal imaging of the fallopian tube by trans-uterine fibreoptic endoscopy as an outpatient procedure. Br J Obstet Gynaecol 1992; 99: 829-835.

[5] Wiedemann R, Sterzik K, Gombisch V, et al. Beyond recanalizing proximal tube occlusion: the argument for further diagnosis and classification. Hum Reprod 1996; 11: 986-91.

[6] Woolcott R. Proximal tubal occlusion: a practical approach. Hum Reprod 1996; 11: 1831-1833.

[7] Woolcott R, Petchpud A, O'Donnell P, et al. Differential impact on pregnancy rate of selective salpingography, tubal catheterization and wire-guide recanalization in the treatment of proximal fallopian tube obstruction. Hum Reprod 1995; 10: 1423-1426.

[8] Dubuisson JB, Chapron C, Ansquer Y, et al. Proximal tubal occlusion: is there an alternative to microsurgery? Hum Reprod 1997; 12: 692-698.

[9] Miettinen A, Heinonen PK, Teisala K, et al. Serologic evidence for the role of Chlamydia trachomatis, Neisseria gonorrhoeae, and Mycoplasma hominis in the etiology of tubal factor infertility and ectopic pregnancy. Sex Transm Dis 1990; 17: 10-14.

[10] Garland SM, Lees MI, Skurrie IJ. Chlamydia trachomatis--role in tubal infertility. Aust N Z J Obstet Gynaecol 1990; 30: 83-86.

[11] Cetin MT, Vardar MA, Aridogan N, et al. Role of Chlamydia trachomatis infections in infertility due to tubal factor. Indian $\mathrm{J}$ Med Res 1992; 95: 139-143.

[12] Paavonen J, Eggert-Kruse W. Chlamydia trachomatis: impact on human reproduction. Hum Reprod Update 1999; 5: 433-447.

[13] de Barbeyrac B, Papaxanthos-Roche A, Mathieu C, et al. Chlamydia trachomatis in subfertile couples undergoing an in vitro fertilization program: a prospective study. Eur J Obstet Gynecol Reprod Biol 2006; 129: 46-53.

[14] Torode HW, Wheeler PA, Saunders DM, et al. The role of chlamydial antibodies in an in vitro fertilization program. Fertil Steril 1987; 48: 987-990. 